\title{
BENEFÍCIOS DA SIMULAÇÃO DINÂMICA NA INDÚSTRIA MINERAL: UTILIZANDO O SIMULADOR DE PROCESSOS DINÂMICO IDEAS PARA VALIDAÇÃO DE FLUXOGRAMAS DE ENGENHARIA*
}

Henrique Paier Milanez ${ }^{1}$ Rafael Villela Ferreira ${ }^{2}$

Savio Vargas Lorentz ${ }^{2}$

\section{Resumo}

À medida que os depósitos minerais de alta qualidade vão se exaurindo e a contínua busca por menores custos operacionais aumentando, as rotas de beneficiamento de minério tornam-se mais complexas, gerando custos de capital cada vez maiores. Dessa forma, surgem desafios não somente para a redução desses custos, mas também para validação do dimensionamento dos equipamentos, teste das lógicas de controle, treinamento operacional e redução do tempo de start-up. No presente trabalho é realizada uma breve revisão bibliográfica sobre o atual cenário de simulação de processos na indústria mineral e é mostrado como essa tecnologia se encaixa em todas as etapas do desenvolvimento de projetos. Especificamente, são apresentados estudos de dimensionamento de linhas de processo e dimensionamento de bombas centrífugas em casos técnicos em que o software IDEAS foi utilizado como ferramenta de simulação dinâmica para validação do processo, reduzindo o impacto financeiro em etapas posteriores. Como exemplo dos resultados das validações, mais de 40 bombas tiveram seu dimensionamento reavaliado e mais de 10 tubulações tiveram seus diâmetros revisados. Os resultados observados corroboram o grande potencial tanto da ferramenta computacional, quanto da simulação de processos de modo geral.

Palavras-chave: IDEAS; Simulação dinâmica; Mineração; Validação de processo.

\section{BENEFITS OF DYNAMIC SIMULATION FOR MINERAL INDUSTRY: VALIDATING ENGINEERING DIAGRAMS WITH IDEAS DYNAMIC SIMULATOR}

\section{Abstract}

As the mineral deposits in terms of quality become less abundant and the pressure for low operational costs increases, the process routes become more complex, generating higher capital costs. Therefore, arises not only the challenges for the capital cost reduction, but also for design validation, control logic testing, operational training and start-up time reduction. This paper will present a brief review of the current scenario of process simulation on mineral industry and how this technology can fit in every project stage. Specifically will be presented a study of process lines and centrifugal pumps design in technical cases where IDEAS software has been used as a dynamic simulation tool for process validation, reducing the financial impact on further project stages. As an example of the results, more than 40 pumps had their design revaluated and more than 10 lines had their diameter reviewed. The results show the great potential of the computational tool as well as the process simulation altogether.

Keywords: IDEAS; Dynamic Simulation; Mining; Process Validation. 


\section{INTRODUÇÃO}

\subsection{Simulação de Processos}

De acordo com Ferreira [1], o termo "modelo" é utilizado para definir o conjunto de equações matemáticas capazes de descrever um sistema real. Um sistema, por sua vez, pode ser definido como uma coletânea de estruturas e recursos que interagem segundo uma determinada lógica de modo que seja possível alcançar um ou mais objetivos. A simulação, pois, é uma das ferramentas mais modernas para projetar e analisar o desempenho de sistemas e de processos complexos.

Garcia [2] afirma que a simulação de sistemas é uma poderosa ferramenta de suporte para a investigação de problemas tipicamente observados na área da engenharia de processos industriais. Ela pode ser usada desde a fase de projeto até a operação de plantas, incluindo as fases de análise de viabilidade econômica do processo. Na Tabela 1 podem ser identificadas algumas aplicações da simulação a um dado processo.

Tabela 1. Aplicações da simulação de processos

\begin{tabular}{|c|c|}
\hline Desafios do Processo & Aplicação da Simulação \\
\hline Projeto de Equipamentos & $\begin{array}{l}\text { - Explorar o dimensionamento/arranjo } \\
\text { físico de equipamentos } \\
\text { - Estudar a interação de várias partes } \\
\text { do processo }\end{array}$ \\
\hline Operação da Planta & $\begin{array}{l}\text { - Melhorar o entendimento do processo } \\
\text { - Desenvolver, testar e qualificar } \\
\text { procedimentos operacionais de } \\
\text { partida, parada e testes } \\
\text { - Analisar diferentes modos ou } \\
\text { filosofias de operação } \\
\text { - Prover recursos para treinamento de } \\
\text { operadores nas mais diversas } \\
\text { condições operacionais }\end{array}$ \\
\hline Sistemas de Controle de Processos & $\begin{array}{l}\text { - Selecionar ajustes de controladores, } \\
\text { projetar lógicas de controle }\end{array}$ \\
\hline Otimização das Condições Operacionais & $\begin{array}{l}\text { - Melhorar a utilização dos recursos } \\
\text { disponíveis } \\
\text { - Maximizar rendimentos operacionais } \\
\text { e minimizar custos }\end{array}$ \\
\hline
\end{tabular}

*Adaptada de Garcia [2]

O desenvolvimento de modelos matemáticos voltados à simulação de processos fundamenta-se em um método interativo no qual modelos são elaborados, cenários são definidos, testes são realizados e resultados são analisados. A metodologia é composta por estágios específicos, tais como a definição do projeto e seus objetivos, abstração, representação digital, experimentação do modelo e documentação do projeto, como relatado por Centeno [3]. A Figura 1 apresenta um fluxograma proposto por Centeno [3] para as etapas do desenvolvimento de modelos de simulação.

\footnotetext{
* Contribuição técnica ao $18^{\circ}$ Seminário de Automação e TI Industrial, 23 a 26 de setembro de 2014,
} São Paulo, SP, Brasil. 

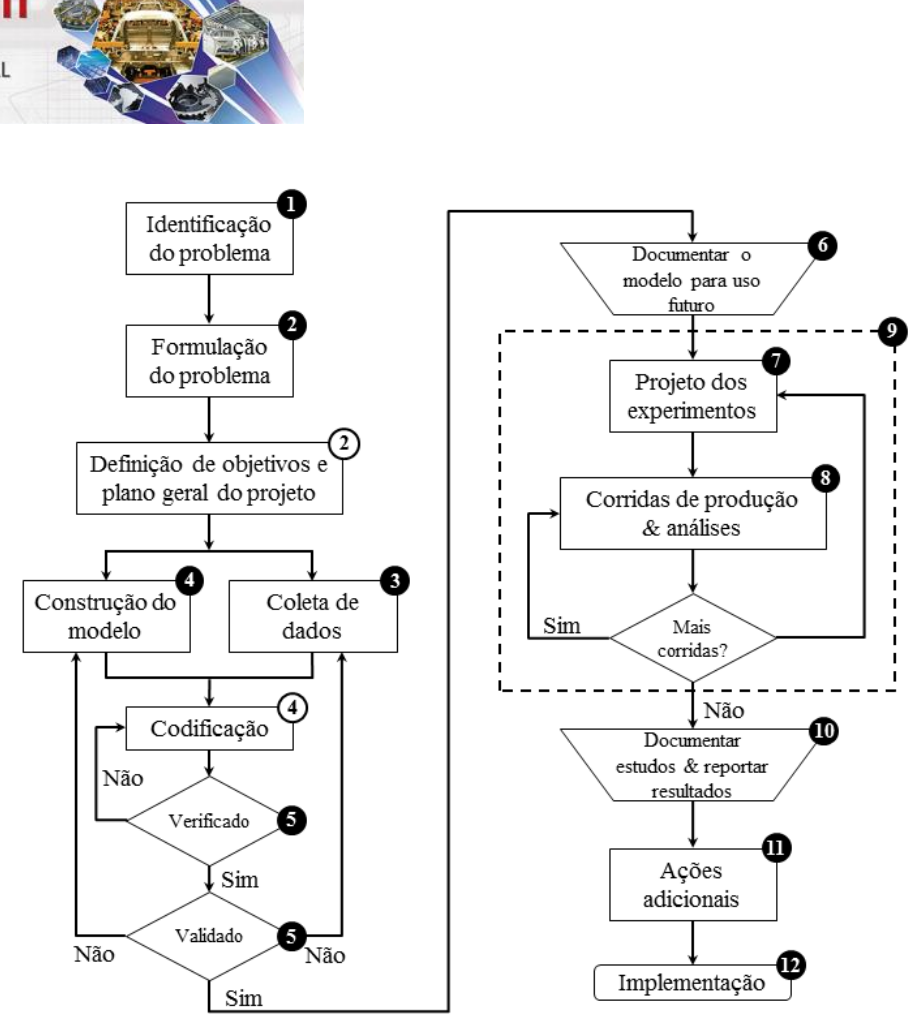

Figura 1. Metodologia de desenvolvimento de modelos de simulação. Adaptado de Centeno [3]

Com o intuito de se assegurar a fidelidade entre o modelo matemático e o sistema real em análise, a verificação e validação do modelo são atividades de grande importância. Os métodos de verificação e validação podem ser informais, estáticos, dinâmicos, simbólicos, restritivos e formais. A descrição completa desses procedimentos pode ser encontrada nos trabalhos de Balci [4-6], Sargent [7] e Centeno [3].

Em linhas gerais, a etapa de verificação corresponde à metodologia para determinar se o modelo funciona conforme o pretendido. Trata-se, portanto, do processo de depuração do modelo computacional realizado a fim de certificar que sua formulação conceitual foi corretamente transcrita através de linguagens de computação, garantido que os balanços de massa, energia e quantidade de movimento sejam atendidos.

A validação do modelo, por sua vez, tem como objetivo realizar a averiguação do modelo proposto quanto à sua capacidade de representar adequadamente (de forma contínua e consistente) o sistema real, assim como descrito por Sargent [7] e Centeno [3]. Devem-se questionar empiricamente as considerações estruturais do modelo e as eventuais suposições acerca dos dados utilizados. Após as etapas de verificação e validação, tem-se um modelo dinâmico preparado para a validação de engenharia.

A ideia de se usar simulação dinâmica para validação de engenharia e para treinamento operacional vem sendo discutida nas últimas décadas. Parthasarathi, Szaruga e Szatkowski [8] relatam que durante o projeto de uma planta, os trabalhos são divididos em múltiplas disciplinas e muitas vezes entre diferentes empresas de engenharia. Visto que cada grupo tende a adicionar um fator de segurança no seu projeto, isso pode levar a significantes superdimensionamentos e, logo, um aumento do custo de capital. Anderson e Nikkhah [9] discutem que ao avançar no projeto, ferramentas de simulação de processos podem transferir os balanços de massa e energia em estado estacionário para uma plataforma dinâmica. Assim, válvulas, tubulações, tanques e bombas podem ser dimensionados e colocados a teste das lógicas de controle e operação.

* Contribuição técnica ao 18Seminário de Automação e TI Industrial, 23 a 26 de setembro de 2014, São Paulo, SP, Brasil. 


\subsection{Simulador de Processos IDEAS}

O simulador de processos IDEAS possui uma interface gráfica baseada em objetos, na qual o usuário monta seu modelo escolhendo objetos de diferentes bibliotecas e os conectando através de linhas de conexão. De acordo com Parthasarathi, Szaruga e Szatkowski [8] IDEAS pode realizar um balanço de massa e energia em estado estacionário, acompanhar fluxo e concentração de componentes, compostos e elementos, identificar variações na distribuição granulométrica, assim como calcular densidade e entalpia. Segundos os autores, operações unitárias simuladas em IDEAS usam princípios elementares, isto é, balanços de massa, energia e momento, configurando a base para o dimensionamento das operações unitárias do processamento mineral. Além dos princípios básicos, IDEAS também possui equações semi-empíricas e correlações específicas para determinadas operações unitárias que não podem ser previstas por equações matemáticas embasadas em princípios físicos fundamentais.

De acordo com Parthasarathi, Parthasarathi, Szaruga e Szatkowski [8] um simulador dinâmico como IDEAS pode ser utilizado em todas as etapas do ciclo de vida de um projeto de uma planta de processamento de minério, isto é, desenvolvimento de processos, validação de engenharia, dimensionamento dos equipamentos, ajuste de malhas de controle, teste das lógicas de controle e treinamento operacional.

O presente trabalho tem por objetivo demonstrar como um simulador dinâmico como - IDEAS vem sendo utilizado para validação de fluxogramas de engenharia utilizando princípios fluidodinâmicos fundamentais e informações de engenharia detalhada. Ainda, será apresentado estudos de casos em que o simulador de processos IDEAS foi fundamental para a avaliação do dimensionamento de linhas e de bombas em determinada etapa do projeto.

\section{MATERIAIS E MÉTODOS}

Um típico projeto de simulação pode ser divido em duas etapas distintas e em subetapas dependendo do objetivo final da simulação. Na Figura 2 são apresentadas estas etapas típicas, conforme a ordem cronológica de realização durante o projeto. No presente trabalho, a validação do processo será o foco principal.

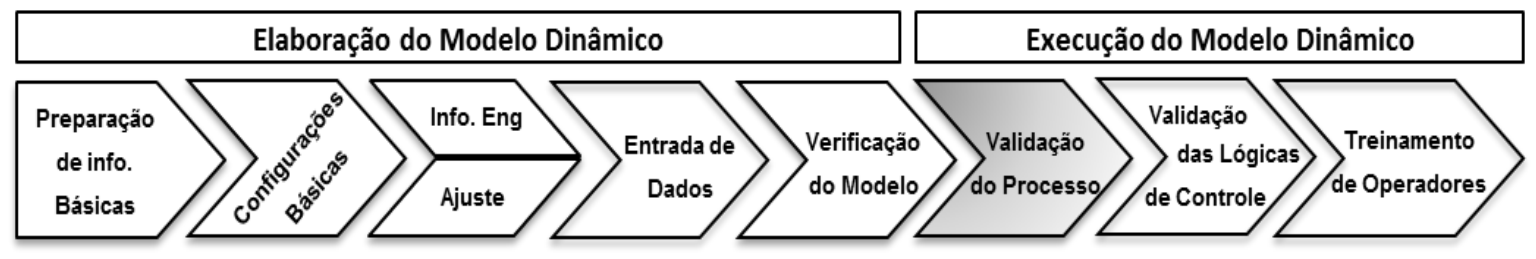

Figura 2 .Ciclo de vida típico de um projeto de simulação de processos

\subsection{Verificação e Validação do Modelo e do Processo}

O principal objetivo desta etapa do processo de construção do modelo é certificar que este está adequado e é capaz de representar o sistema real com relativa fidelidade e significância. É imprescindível neste momento estabelecer os testes aos quais o modelo deve ser submetido.

\footnotetext{
* Contribuição técnica ao $18^{\circ}$ Seminário de Automação e TI Industrial, 23 a 26 de setembro de 2014 , São Paulo, SP, Brasil.
} 
A partir do projeto detalhado, as falhas de projeto podem ser identificadas facilmente em um modelo dinâmico quando comparadas a identificação de falhas em planilhas de cálculo. Por possuir inúmeras entradas de engenharia detalhada, um modelo dinâmico funciona como uma planta virtual e, desta forma, é capaz de gerar resultados conclusivos sobre o dimensionamento de equipamentos, flexibilidade operacional, estratégia de controle, balanço de massa, qualidade do produto entre outros.

A validação de P\&ID nada mais é do que a representação de todos os dados de engenharia detalhada de uma forma integrada. As informações de engenharia são retiradas dos documentos do projeto e são inseridas dentro dos blocos do IDEAS. Conforme Parthasarathi, Szaruga e Szatkowski [8] nenhuma outra sub-rotina ou programação é necessária, já que após colocar todos os dados necessários e conectá-los, os fluxos e as pressões associadas a linha de pressão serão automaticamente calculados. Dessa forma, uma boa representação de um fluxograma de engenharia pode ser simulada em IDEAS, conforme exemplificado na Figura 3. Neste exemplo, fica aparente como todos os equipamentos e malhas de controle do fluxograma são simulados.

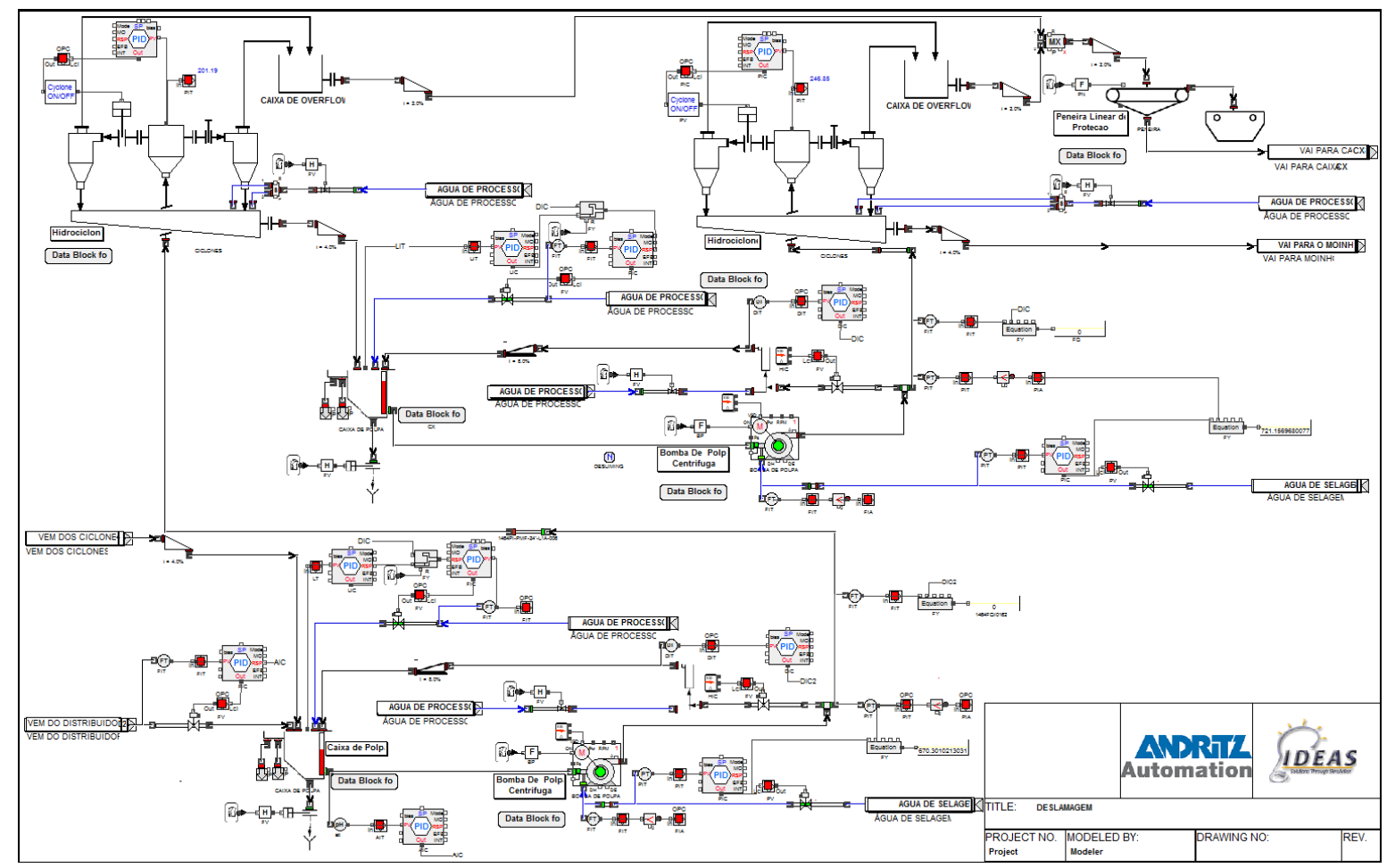

Figura 3 . Exemplo de um fluxograma de engenharia simulado no software IDEAS

\section{RESULTADOS E DISCUSSÃO}

Os casos aqui apresentados foram retirados da experiência dos autores em projetos passados realizados em Belo Horizonte - MG em que a simulação dinâmica foi utilizada para a validação de engenharia de uma grande mineradora. Os casos selecionados foram o dimensionamento de linhas e de bombas. Estes foram avaliados com a ajuda do software IDEAS.

* Contribuição técnica ao 185eminário de Automação e TI Industrial, 23 a 26 de setembro de 2014, São Paulo, SP, Brasil. 


\subsection{Estudo de Casos - Dimensionamento de Linhas}

Um importante item a ser analisado a partir da simulação de processos consiste no dimensionamento de linhas, tanto de polpa quanto de água. Assim, devido ao elevado número de tubulações existentes em todos os tipos de projetos, o dimensionamento adequado dessas linhas é crucial para uma validação adequada do processo. Na Figura 4, é apresentada parte do modelo dinâmico, construído utilizando o software IDEAS, contendo caixa de polpa, bomba de polpa e tubulações de água e de polpa.

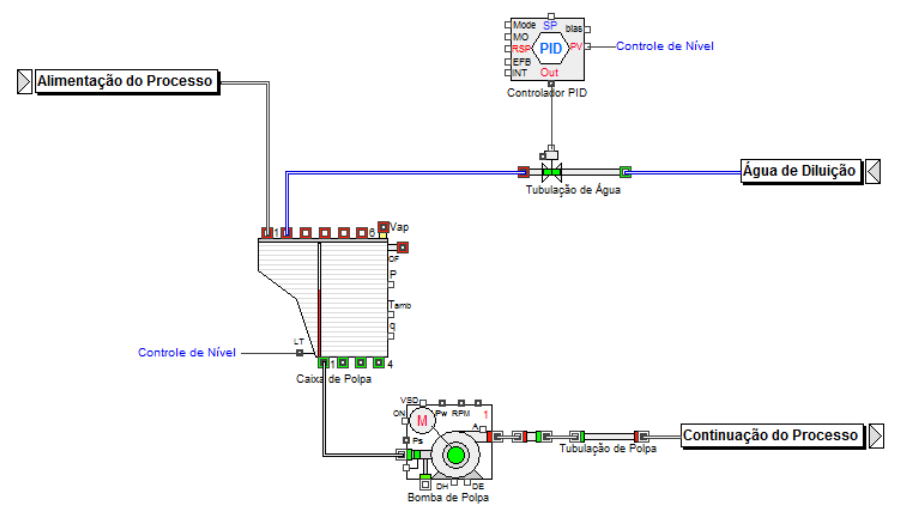

Figura 4.Parte de Modelo Dinâmico, contendo tubulações, em IDEAS

Com o intuito de que o modelo dinâmico represente o processo de forma fidedigna, as informações de engenharia necessárias para os equipamentos devem ser inseridas nos respectivos blocos do software. Como principais dados a serem utilizados, pode-se destacar o comprimento, diâmetro, espessura da tubulação, rugosidade e comprimento equivalente. Desse modo, na Figura 5 são apresentados os campos do objeto Pipe (objeto equivalente à tubulação no software) que devem ser inseridos pelo usuário durante a modelagem.

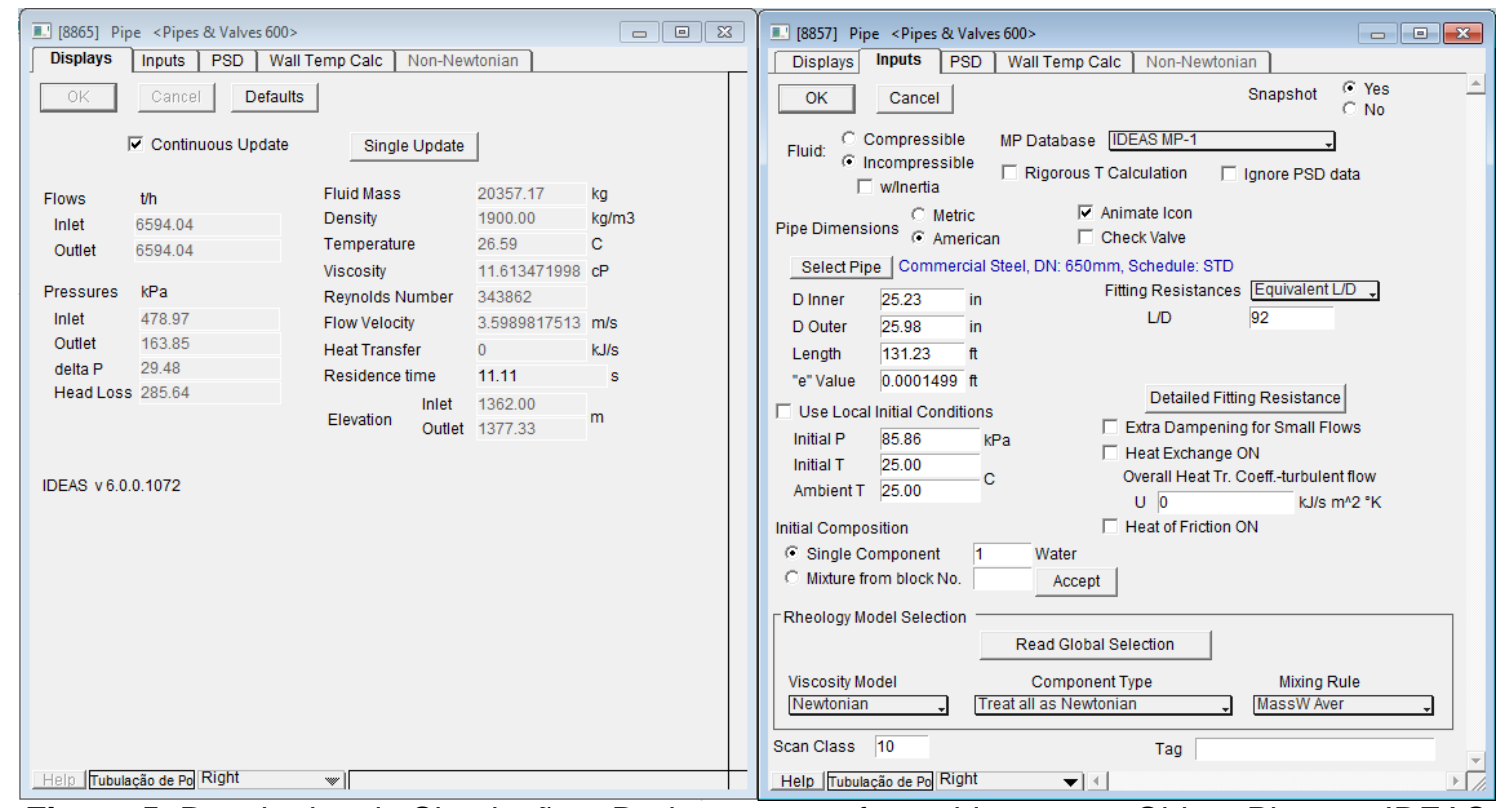

Figura 5. Resultados da Simulação e Dados a serem fornecidos para o Objeto Pipe em IDEAS

* Contribuição técnica ao $18^{\circ}$ Seminário de Automação e TI Industrial, 23 a 26 de setembro de 2014, São Paulo, SP, Brasil. 
$\mathrm{Na}$ simulação de tubulações de polpa, um dos principais problemas passível de análise consiste na sedimentação de sólidos no interior das tubulações. A partir de informações de vazão volumétrica, diâmetro, espessura da tubulação e revestimento da linha, o software calcula o valor da velocidade de transporte da polpa. Esse valor é então comparado com a velocidade crítica de escoamento, ou seja, a velocidade limite para que o escoamento ocorra sem haver sedimentação na tubulação, que é calculada usando a forma modificada da equação de Durand [10].

Assim, durante o estudo de linhas de projetos já realizados, verificou-se que a velocidade de transporte de determinadas linhas apresentava um valor inferior à velocidade crítica calculada, ocasionando a possibilidade de sedimentação no interior da tubulação. Para esses casos, as tubulações foram redimensionadas, tendo seus diâmetros reduzidos, de forma a aumentar as velocidades de escoamento para um valor superior ao valor limite, evitando assim o depósito de material dentro da linha.

Já para o caso da simulação de tubulações de água, busca-se inicialmente avaliar a velocidade de escoamento em relação à velocidade de referência, sendo adotado o intervalo de 2 a $3 \mathrm{~m} / \mathrm{s}$, conforme sugerido por Telles [11]. Adicionalmente, analisa-se a adequação do diâmetro de tubulações de adição de água para o controle de nível de caixas de polpa. De modo geral, a partir de fatores de engenharia que variam entre diferentes empresas projetistas, o controle de nível apenas pode ser realizado de maneira satisfatória caso a linha de água seja capaz de fornecer uma vazão maior que determinada porcentagem da vazão da linha principal de polpa, sendo, por exemplo, comumente utilizado o valor de $30 \%$ da vazão de polpa. Desse modo, para vazões de água menores do que este valor, não seria possível obter um controle de nível adequado, sobretudo quando da necessidade de se aumentar o nível da caixa.

Assim, foram observados, através de simulação dinâmica, sistemas de adição de água de diluição incapazes de fornecer uma vazão considerada suficiente para um controle satisfatório do nível das caixas de polpa, fazendo com que as caixas se esvaziassem e, consequentemente, as bombas cavitassem. Nesses casos, duas abordagens diferentes podem ser seguidas. A primeira consiste em aumentar 0 diâmetro da linha de adição de água, aumentando assim a vazão de água para controle, de modo a, com velocidades coerentes de transporte, permitir um controle efetivo do nível. Já na segunda alternativa, por sua vez, diante da incapacidade do sistema controlar o nível da caixa de polpa de forma adequada, deve-se reavaliar a necessidade do uso de válvulas de controle para essa função, sobretudo para os casos em que as bombas logo à montante possuam inversor de frequência capaz de efetuar o devido controle de nível.

\subsection{Estudo de Casos - Dimensionamento de Bombas}

Os sistemas de bombeamento são bastante analisados e estudados via simulação dinâmica de processos. A criticidade da validação de bombas reside no fato de seu dimensionamento ser interdisciplinar, estando sujeita a modificações devido a alterações de arranjo de equipamentos e tubulações. Desse modo, em projetos previamente realizados, foram encontradas diversas inconsistências em sistemas de bombeamento, que estavam tanto superdimensionados quanto subdimensionados.

Durante o processo de modelagem e validação do sistema de bombeamento, informações de engenharia tanto do fornecedor quanto relativas ao arranjo gerado pela projetista foram inseridas no software. Na Figura 6 são apresentadas as janelas

\footnotetext{
* Contribuição técnica ao $18^{\circ}$ Seminário de Automação e TI Industrial, 23 a 26 de setembro de 2014, São Paulo, SP, Brasil.
} 
do objeto Pump no software IDEAS contendo os resultados e as informações que são utilizadas para os cálculos. Como pode ser verificado, o objeto considera os principais fatores necessários para o dimensionamento de uma bomba de polpa, dentre os quais se destacam a curva da bomba, curva para vazão máxima, curva para NPSH (Net Positive Suction Head), a rotação e possíveis perdas de eficiência. Também é importante ressaltar que o objeto efetua o cálculo de toda a rede de pressão do bombeamento, considerando a pressão de entrada dos equipamentos a jusante, os dados relativos à tubulação de recalque e a elevação dos equipamentos a montante e a jusante.

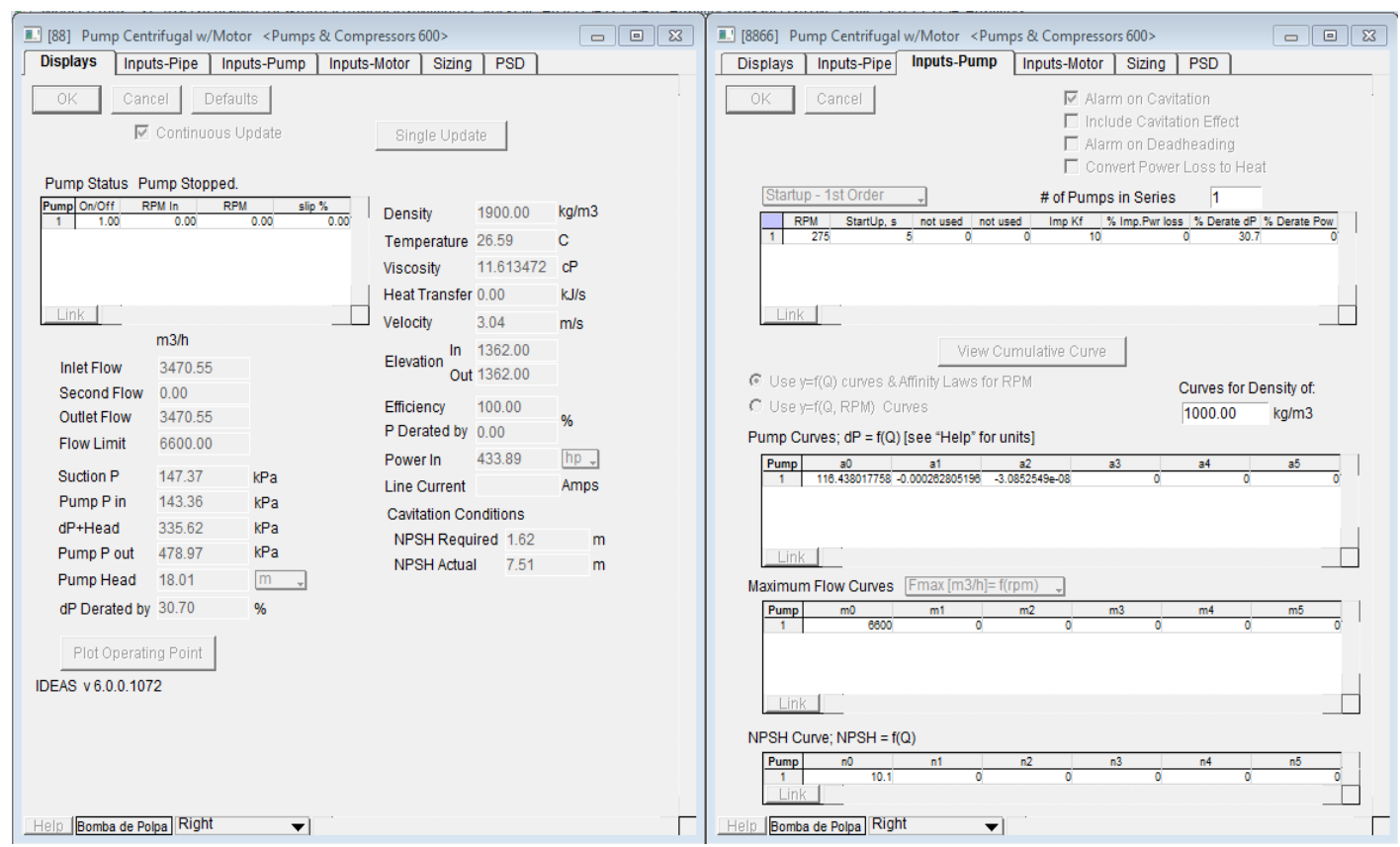

Figura 6. Resultados da Simulação e Dados a serem fornecidos para o Objeto Pipe em IDEAS

Para facilitar o processo de inserir as curvas das bombas no software, é utilizado um objeto denominado Graph Digitalizer, que, a partir do desenho das curvas, é capaz de efetuar uma regressão e obter a equação da curva correspondente à folha de dados do fornecedor. Na Figura 7 é ilustrada as representações da curva do fornecedor e sua representação no IDEAS.
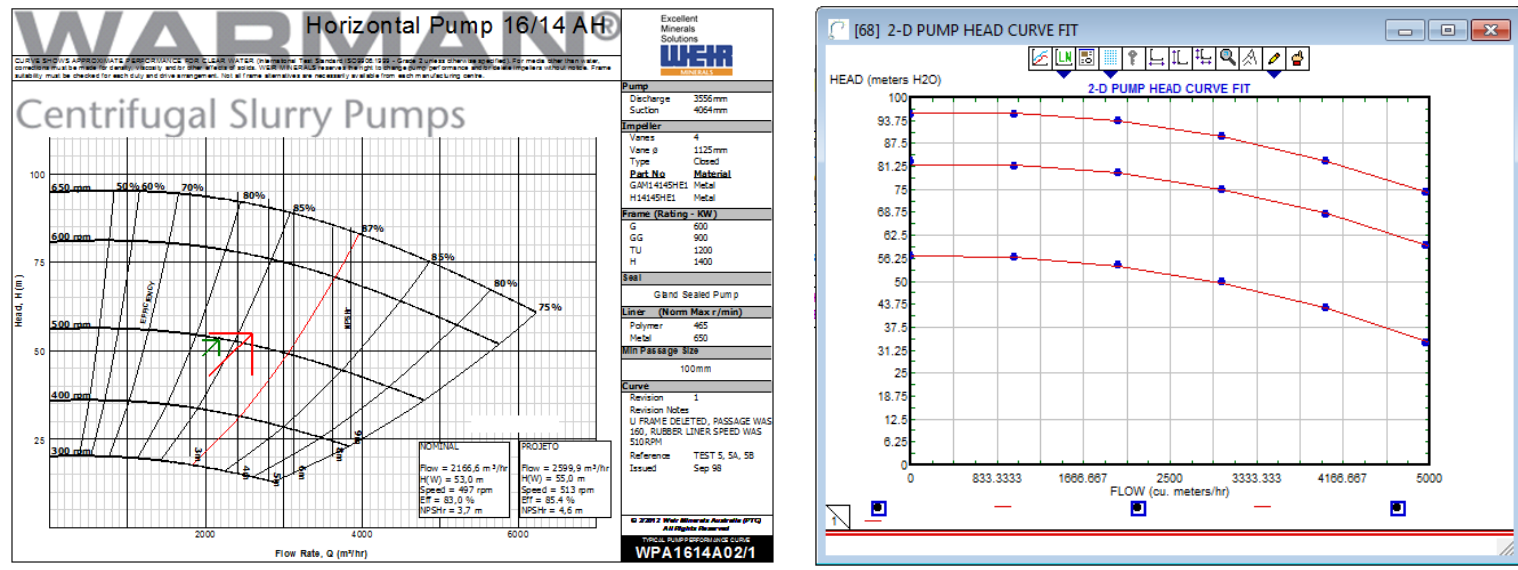

Figura 7. Representação da curva de bomba do fornecedor [12] no software IDEAS

* Contribuição técnica ao 18Seminário de Automação e TI Industrial, 23 a 26 de setembro de 2014, São Paulo, SP, Brasil. 
A partir de simulação dinâmica de processos, foram revisados diversos sistemas de bombeando, consistindo assim em um dos principais benefícios verificados com a validação de processos. De modo geral, podem-se verificar, via simulação, três principais problemas relativos aos sistemas de bombeamento: diluição excessiva da polpa devido a controle de nível, caixa de polpa transbordando ou esvaziando. Nesses três casos, a partir de uma perturbação no processo, é possível visualizar problemas no dimensionamento do sistema de bombeamento. Caso seja verificado que a caixa de polpa esteja esvaziando ou que a polpa esteja sendo demasiadamente diluída devido à adição em excesso de água para controle de nível, conclui-se que a bomba em questão está superdimensionada. Por outro lado, transbordos em caixas de polpa associadas a sistemas de bombeamento demonstram que a bomba simulada não apresenta capacidade de bombear toda a vazão requerida, estando assim subdimensionada.

De modo geral, esses fenômenos verificados em simulação que possibilitam a visualização de um dimensionamento inadequado de bombas ocorrem devido a modificações comuns em projetos, mas que, quando não associadas com a respectiva revisão das bombas impactadas, tornam-se erros de projeto. Dentre as três principais causas de dimensionamento inadequado de sistemas de bombeamento, conforme verificado em projetos previamente realizados, destacamse três casos: a retirada de inversor de frequência, alterações na documentação de projeto (como arranjos e especificações de equipamentos a jusante) e aplicação excessiva de fatores de projeto (margens de segurança).

No primeiro caso, foram encontradas situações em que, durante a fase de engenharia detalhada, com o intuito de reduzir investimentos em bens de capital (CAPEX), foi decidido não adquirir os inversores de frequência de algumas bombas consideradas de menor criticidade para o processo. Entretanto, ao se retirar os inversores de frequência, as bombas, que antes trabalhavam com a rotação definida como nominal, passaram a operar com a rotação de projeto, podendo gerar vazões de $20 \%$ a $40 \%$ superiores. Desse modo, o dimensionamento dessas bombas deveria ter sido devidamente revisado, quando da retirada dos inversores. Como isso não havia sido feito, essas bombas estavam gerando, na simulação, resultados de vazão $20 \%$ superiores ao balanço de massa, gerando impactos em toda a análise do processo. Assim, para as caixas de polpa que possuíam adição de água para controle de nível, foi necessário adicionar uma maior vazão de água de diluição, ocasionando a diluição excessiva da polpa. Já para as caixas de polpa sem controle de nível, verificou-se o esvaziamento dessas, com a consequente cavitação das bombas a jusante.

No segundo caso, foram encontradas situações em que alterações nos arranjos mecânicos ou na especificação dos equipamentos a jusante, decorrentes de modificações inerentes ao projeto, resultaram em erros de projeto. Isso ocorreu devido ao fato de essas mudanças terem sido realizadas após o dimensionamento das bombas, sem que essas fossem posteriormente revisitadas. Desse modo, alterações no arranjo geraram significativas variações na altura manométrica total das bombas, devido, sobretudo, a mudanças no desnível geométrico e aumento da perda de carga total. Assim, alterações de elevação, quantidade de acidentes e comprimento de tubulação podem acarretar na inadequação do sistema de bombeamento, de modo que o dimensionamento das respectivas bombas deve ser revisado.

\footnotetext{
* Contribuição técnica ao $18^{\circ}$ Seminário de Automação e TI Industrial, 23 a 26 de setembro de 2014, São Paulo, SP, Brasil.
} 


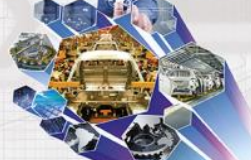

Dessa forma, existiram casos em que as alterações observadas em projeto acarretaram o aumento da altura manométrica total, com 0 consequente subdimensionamento do sistema previamente projetado, enquanto em outros esse parâmetro acabou sendo reduzido, resultando em bombas superdimensionadas. Para ambos os casos, foram visualizados, durante a simulação, fenômenos, tais como diluição excessiva de polpa, transbordo e esvaziamento de tanques, que impactariam não apenas o funcionamento do processo em questão, mas sim a partida e operação da planta como um todo.

Além disso, foram também encontrados erros no dimensionamento de bombas decorrentes da inconsistência ou modificação da pressão residual de equipamentos a jusante, como, por exemplo, a pressão de alimentação de hidrociclones. Assim, alterações na pressão de entrada dos hidrociclones, situados logo após o sistema de bombeamento, não foram consideradas para a revisão das especificações das bombas, acarretando em alterações do ponto de operação e os consequentes prejuízos ao processo. Isso evidencia uma das principais vantagens da simulação dinâmica de processos, que permite uma validação do processo como um todo, não apenas de cada equipamento de maneira isolada, facilitando a visualização de inconsistências entre as especificações dos equipamentos de um sistema.

Por fim, para o terceiro caso, foram também encontradas situações relacionadas à adoção de fatores de segurança em cima de fatores de segurança previamente aplicados, acarretando em uma especificação final significativamente superior ao necessário para atender ao processo. Por exemplo, foram encontrados casos de superdimensionamento em bombas sem inversor de frequência, que, muitas vezes, de acordo com premissas da projetista, já são especificadas para prover uma vazão de projeto $20 \%$ superior ao valor nominal, presente no balanço de massa. Além disso, para o dimensionamento dessas bombas também havia sido considerado, como margem de segurança, um valor de pressão residual superior à pressão de operação dos equipamentos a jusante. Feito isso, foram selecionadas polias ou rotores, devido às especificações padrão dos fornecedores, diferentes do valor calculado, contribuindo para o superdimensionamento do conjunto motor-bomba em questão. Portanto, ao se computar essas três fontes de superdimensionamento, teve-se como resultado um sistema de bombeamento significativamente superdimensionado, gerando o constante esvaziamento da caixa de polpa e a prejudicando a continuidade do processo.

\section{CONCLUSÃO}

A simulação dinâmica de processos ainda é muito pouco familiar à mineração e isso impede, muitas vezes, por limitações humanas ou de softwares não tão robustos, uma análise mais detalhada do processo durante toda a vida do projeto. Os ganhos obtidos através da simulação dinâmica são rápidos e a tomada de decisão, com informações embasadas em teorias já amplamente aceitas para o dimensionamento de equipamentos, torna-se bem mais simples e rápida.

O presente trabalho descreveu como uma ferramenta como o IDEAS, que se mostra a ser uma ferramenta simples e robusta, pode vir a se tornar uma importante ferramenta em mineração. Os casos técnicos apresentados mostraram que no total, somente se atentando para erros no dimensionamento de bombas e de linhas, foram encontrados mais de 50 potenciais problemas no projeto simplesmente inserindo as informações de engenharia no software e acompanhando os resultados fornecidos pelo mesmo.

\footnotetext{
* Contribuição técnica ao $18^{\circ}$ Seminário de Automação e TI Industrial, 23 a 26 de setembro de 2014, São Paulo, SP, Brasil.
} 
Assim, fica claro o grande potencial tanto do software IDEAS quanto da simulação de processos para a indústria mineral como um todo, visto que os potenciais ganhos são ainda pouco explorados e as oportunidades de negócios, muito grandes.

\section{REFERÊNCIAS}

1 Ferreira RV. Desenvolvimento de uma Metodologia para a Simulação Dinâmica do Processo de Purificação de Hidrogênio. 2013. 188f. Dissertação (Mestrado em Engenharia Química) - Universidade Federal de Minas Gerais, Belo Horizonte, 2013.

2 Garcia C. Modelagem e simulação de processos industriais e de sistemas eletromecânicos.2.ed. São Paulo: EDUSP, 2005. 678 p.

3 Centeno MA. An introduction to simulation modeling. In: IEEE Computer

4 Balci $O$. Golden rules of verification, validation, testing, and certification of modeling and simulation applications. no, v. 4, 2010.

5 Balci O. Verification, validation, and accreditation. In: IEEE Computer Society Press. Proceedings of the 30th conference on Winter simulation. [S.I.], 1998.

6 Balci, O. Principles and techniques of simulation validation, verification, and testing. In: IEEE. Simulation Conference Proceedings, 1995. Winter. [S.I.], 1995. p.147-154.

7 Sargent, R. G. Verification and validation of simulation models. In: Winter Simulation Conference. Proceedings of the 37th conference on Winter simulation. [S.I.], 2005. p. 130-143.

8 Parthasarathi P, Szaruga V, Szatkowski M. Benefits of Dynamic Process Simulation for Mineral. 2009. [acesso em 23 mai. 2014]. Disponível em http://www.andritz.com/aaautomation-benefits-dynamicprocesssimulation-mineral.pdf.

9 Anderson C, Nikkhah K. Role of Simulation Software in the Design and Operation of Metallurgical. 2001. [acesso em 23 mai. 2014]. Disponível em http://www.andritz.com/ja/aa-automation-simulation-metallurgical-plants.pdf

10 Durand R. The Hydraulic transportation of coal and solid materials in pipes. London colloquium of the national coal board. 1952.

11 Telles PCS. Tabelas e gráficos para projetos de tubulações. Pedro Carlos da Silva Telles DGPB. 6 ED. Ver. E ampliada. Rio de janeiro: interciência. 1998.

12 Weir Minerals Software. Versão wsCAT® 4.0, 2008.

* Contribuição técnica ao $18^{\circ}$ Seminário de Automação e TI Industrial, 23 a 26 de setembro de 2014, São Paulo, SP, Brasil. 